\title{
Ab initio LCAO study of the atomic, electronic and magnetic structures and the lattice dynamics of triclinic $\mathrm{CuWO}_{4}$
}

\author{
A. Kuzmin ${ }^{\mathrm{a}, *}$, A. Kalinko ${ }^{\mathrm{a}}$, R. A. Evarestov ${ }^{\mathrm{b}}$ \\ ${ }^{a}$ Institute of Solid State Physics, University of Latvia, Kengaraga Street 8, LV-1063 \\ Riga, Latvia \\ ${ }^{b}$ Department of Quantum Chemistry, St.Petersburg State University, 26 Universitetskiy \\ Prospekt, Stary Peterhof 198504, Russia
}

\begin{abstract}
The electronic, structural and phonon properties of antiferromagnetic triclinic $\mathrm{CuWO}_{4}$ have been studied using the first-principle spin-polarised linear combination of atomic orbital (LCAO) calculations based on the hybrid exchange-correlation density functional (DFT)/Hartree-Fock (HF) scheme. In addition, the local atomic structure around both $\mathrm{Cu}$ and $\mathrm{W}$ atoms has been probed using the extended X-ray absorption fine structure (EXAFS) spectroscopy. We show that by using the hybrid DFT-HF functional, one can accurately and simultaneously describe the atomic structure (the unit cell parameters and the atomic fractional coordinates), the band gap and the phonon frequencies. In agreement with our EXAFS results, the LCAO calculations reproduce a strong distortion of both the $\mathrm{CuO}_{6}$ and the $\mathrm{WO}_{6}$ octahedra, which occur due to the first-order and second-order Jahn-Teller
\end{abstract}

\footnotetext{
*Corresponding author

Email address: a.kuzmin@cfi.lu.lv (A. Kuzmin)

URL: http://www.cfi.lv/ (A. Kuzmin)
} 
effects, respectively. We found that the HF admixture of $13-16 \%$, which is implemented in the PBE0-13\% and WCGGA-PBE-16\% functionals, produces the best result for $\mathrm{CuWO}_{4}$. The calculated properties agree well with the available experimental data provided by diffraction, optical, X-ray photoelectron and Raman spectroscopies.

Keywords: Copper tungstate, Antiferromagnetic, Ab initio calculations, Electronic band structure, Lattice dynamics

\section{Introduction}

Earlier studies $[1,2,3,4,5]$ of triclinic copper tungstate $\mathrm{CuWO}_{4}$ were motivated by its magnetic properties, which were determined by the paramagneticto-antiferromagnetic transition below the Néel temperature $T_{\mathrm{N}}=24 \mathrm{~K}$ [6]. The ground state of $\mathrm{Cu}^{2+}$ ions is almost purely $S=1 / 2$, which simplifies the theoretical analysis and makes copper tungstate a convenient compound to study the nature of antiferromagnetic ordering despite the low symmetry of its crystallographic lattice $[1,2,3]$. Due to a strong electron-lattice coupling, the low (triclinic) lattice symmetry forms and produces the first-order Jahn-Teller (FOJT) distortion of the $\mathrm{CuO}_{6}$ octahedra and the second-order Jahn-Teller (SOJT) distortion of the $\mathrm{WO}_{6}$ octahedra, which are caused by the $3 d^{9}$ electron configuration of the $\mathrm{Cu}^{2+}$ ions $[7,8,9]$ and a mixing of the empty $5 d$ orbitals in the $\mathrm{W}^{6+}$ ions with the filled $2 p$ orbitals in the oxygen atoms $[10]$.

In recent years, copper tungstate has attracted an increasing interest from the research community because of its numerous possible applications. With a band gap of approximately $2.0-2.3 \mathrm{eV}$ in the thin-film form, $\mathrm{CuWO}_{4}$ 
has been suggested as a photoanode material candidate for the photovoltaic electrochemical (PVEC) cell $[11,12]$. The all-solid-state thin-film lithium batteries, which are based on the nano-sized $\mathrm{CuWO}_{4}$ positive electrode as proposed in [13], show a high-volume rate capacity in the first discharge and lack the unfavourable electrochemical degradation, observed in liquid electrolyte systems [13].

Upon daylight illumination, the non-crystalline $\mathrm{CuWO}_{4}$ has been found to have a significantly higher photocatalytic activity than the reference $\mathrm{TiO}_{2}$ (Degussa P25) when methylene blue experiences the photocatalytic degradation process in neutral water [14]. This result has been explained by the intense light absorption in the blue and yellow-red spectral ranges caused by the electronic structure of copper tungstate [14].

The catalytic activity of $\mathrm{CuWO}_{4}$ can be modified by doping with other $3 d$ ions. In particular, the $\mathrm{Ni}^{2+}$-substituted $\mathrm{Cu}_{0.4} \mathrm{Ni}_{0.6} \mathrm{WO}_{4}$ nanoparticles/nanorods show a better electrocatalytic activity towards the hydrogen evolution reaction than the native $\mathrm{CuWO}_{4}$ and $\mathrm{NiWO}_{4}$ [15]. The effect of the composition on the catalytic activity for carbon monoxide oxidation has been investigated using $\mathrm{Cu}_{1-x} \mathrm{Co}_{x} \mathrm{WO}_{4}[16]$.

The response to carbon monoxide, methane, ammonia and water of gassensitive resistors based on $\left(\mathrm{CuWO}_{4}\right)_{x}\left([\mathrm{SnTi}] \mathrm{O}_{2}\right)_{1-x}$ has been studied in [17]. The observed resistivity behaviour has been interpreted as a result of the partially occupied $e_{g}$ states of the $\mathrm{Cu}^{2+}$ cations, which act as donors and cause an n-type conductivity [17].

A small indirect band gap $(2.0 \mathrm{eV}[11], 2.25 \mathrm{eV}[12,18], 2.3 \mathrm{eV}[19])$ and a high stability against photo-degradation in water [18] make the mesoporous 
polycrystalline $\mathrm{CuWO}_{4}$ thin film a promising candidate for an efficient water splitting photocatalyst.

Thus, to understand the functional properties of copper tungstate and to further optimise them, we must know its precise electronic structure.

To the best of our knowledge, until now, the band structure of $\mathrm{CuWO}_{4}$ has been theoretically studied using only the full-potential linearised augmented plane wave (FP-LAPW) method by two groups [20, 21] and [22]. Although both groups have used the same FP-LAPW method, different values of the band gap have been found. The gap value of $1.9 \mathrm{eV}$ has been obtained in [20, 21], which is unusually large for the employed exchange-correlation potential within the generalised-gradient approximation (GGA), whereas in [22], the energy gaps of $E_{g} \sim 0.54 \mathrm{eV}$ and $\sim 1.5 \mathrm{eV}$ have been found within the local spin density approximation (LSDA) and the local density approximation with the added Hubbard-type Coulomb correction $(\mathrm{LDA}+U)$, respectively. Note that in these works, the calculations have been performed for an experimental $\mathrm{CuWO}_{4}$ structure without structure optimisation.

The dependence of the lattice dynamics and the crystal volume on the pressure in $\mathrm{CuWO}_{4}$ has been studied in [7] within the framework of the density-functional theory (DFT) and the plane-wave pseudopotential method using the LDA and GGA exchange and correlation functionals. A structural phase transition to a monoclinic wolframite-type structure was found at $10 \mathrm{GPa}$ [7], which caused a quenching of the FOJT distortion in the $\mathrm{CuO}_{6}$ octahedra.

In the present work, the atomic, electronic and magnetic structures and the phonon properties of $\mathrm{CuWO}_{4}$ have been systematically studied using the 
first-principle spin-polarised periodic linear combination of atomic orbital (LCAO) method [23] with the pure density functional (DFT) and the hybrid DFT/Hartree-Fock functionals. The obtained results are compared with the results from previous theoretical studies and the available experimental data.

\section{Computational details}

In the present work, the electronic, structural and phonon properties of $\mathrm{CuWO}_{4}$ have been studied using the first-principle spin-polarised LCAO calculations performed using the CRYSTAL09 code [24]. The core electrons of the tungsten and copper atoms were excluded from consideration using the Hay-Wadt (HW) effective small-core pseudopotentials (ECP) and the corresponding atomic basis sets, which exclude any diffuse-Gaussian-type orbitals with the exponents less than 0.1. The basis set for tungsten was optimised by us for $\mathrm{ZnWO}_{4}$ [25], whereas the basis set for copper was taken from [26]. For the oxygen atoms, we used the all-electron basis set, which was optimised in earlier calculations of perovskites [27] and previously used by us in $\mathrm{ZnWO}_{4}[25,28]$ and $\mathrm{NiWO}_{4}$ [29].

In the CRYSTAL09 code [24], the accuracy in evaluating the Coulomb series and the exchange series is controlled by a set of tolerances, which were taken to be $\left(10^{-8}, 10^{-8}, 10^{-8}, 10^{-8}, 10^{-16}\right)$. The Monkhorst-Pack scheme [30] for an $8 \times 8 \times 8 k$-point mesh in the Brillouin zone was applied. The SCF calculations were performed for three pure DFT (LDA [31], PWGGA [32], PBE [33]) and three hybrid DFT-HF (PBE0-13\% \& PBE0-25\% [34], WCGGA-PBE-16\% [35]) functionals. The percentages $13 \%, 16 \%$ and $25 \%$ define the Hartree-Fock admixture in the exchange part of DFT functional. 
$\mathrm{CuWO}_{4}$ is paramagnetic at room temperature. However, it exhibits a long-range antiferromagnetic (AFM) order below its transition temperature of $T_{\mathrm{N}}=24 \mathrm{~K}[2,6]$. In the AFM phase, the magnetic unit cell $(2 a, b, c)$ is twice as large as the original cell along the $a$-axis $[2,6]$. The calculations have been performed for non-magnetic (NM), antiferromagnetic (AFM) and ferromagnetic (FM) states. The total spin projection was fixed in AFM $\left(S_{z}=0\right)$ and $\mathrm{FM}\left(S_{z}=1\right)$ states. In the AFM state, the spins at the two equivalent copper ions within the unit cell order ferromagnetically along the $c$-axis but antiferromagnetically along the $a$-axis $[2,6]$.

The results of our LCAO calculations, which correspond to the lowest temperature limit $(T=0 \mathrm{~K})$, agree with the experimental findings $[2$, 6]. By comparing the total energy per formula unit $E_{\text {tot }}$ for the AFM ($54648.567 \mathrm{eV})$, FM $(-54648.551 \mathrm{eV})$ and NM (-54647.980 eV) phases, which were obtained from the LCAO calculations for the PBE0-13\% functional, we found that the AFM phase has the lowest energy although the small difference is only tenths of the electronvolt range.

The structural parameters such as lattice parameters $(a, b, c, \alpha, \beta, \gamma)$, unit cell volume $\left(V_{0}\right)$ and atomic fractional coordinates $(x, y, z)$, which were calculated for the AFM state using pure DFT and hybrid DFT-HF functionals, are reported in Table 1.

The bond lengths $R(\mathrm{Cu}-\mathrm{O})$ and $R(\mathrm{~W}-\mathrm{O})$ in the $\mathrm{CuO}_{6}$ and $\mathrm{WO}_{6}$ octahedra, which were calculated from the low-temperature experimental [2] and the LCAO (with hybrid DFT-HF WCGGA-PBE-16\% functional) sets of structural parameters, are reported in Table 2. The mean values $\langle R(\mathrm{Cu}-\mathrm{O})\rangle$ and $\langle R(\mathrm{~W}-\mathrm{O})\rangle$ of the bond lengths and their standard deviations $\sigma(\mathrm{Cu}-\mathrm{O})$ 
and $\sigma(\mathrm{W}-\mathrm{O})$, which are used as an estimate of the octahedron distortion, are also given in Table 2.

The bulk modulus $B_{0}$ and its pressure derivative at zero pressure $B_{0}^{\prime}$ were evaluated from a dependence of the total energy on the lattice volume $E(V)$. The internal coordinates and the lattice parameters were optimised while keeping the volume constant, and the energy-versus-volume results were curve-fitted to the second-order Murnaghan equation of state (EOS) [36]. The calculated $B_{0}$ and $B_{0}^{\prime}$ values are reported in Table 1 for the pure DFT (PBE) and hybrid DFT-HF (PBE0-13\%) functionals.

The phonon frequencies at the centre of the Brillouin zone (the $\Gamma$-point) in the AFM phase were calculated using the direct (frozen-phonon) method $[24,37]$. Their values for the three hybrid DFT-HF functionals (PBE0-13\%, WCGGA-PBE-16\% and PBE0-25\%) are reported in Tables 3 and 4 for the Raman-active modes and the infrared (IR)-active modes, respectively.

\section{Results and discussion}

\subsection{Atomic structure}

$\mathrm{CuWO}_{4}$ belongs to a series of structurally related materials $\mathrm{MeWO}_{4}$ $(\mathrm{Me}=\mathrm{Mg}, \mathrm{Mn}, \mathrm{Fe}, \mathrm{Ni}, \mathrm{Co}, \mathrm{Cu}, \mathrm{Zn}, \mathrm{Cd})$, where metal cations and tungsten ions occupy non-equivalent octahedral sites. However, unlike the other tungstates that adopt the wolframite-type structure, $\mathrm{CuWO}_{4}$ has a triclinic crystal structure (space group $P \overline{1}$ (No. 2)) [38]. In this structure, the metaloxygen Jahn-Teller distorted octahedra $\left(\mathrm{CuO}_{6}\right.$ and $\left.\mathrm{WO}_{6}\right)$ of one type share edges and form zigzag chains along the $c$-axis (Fig. 1). The zigzag chains are arranged in alternating layers that are perpendicular to the $a$-axis direction. 
The lattice parameters and atomic fractional coordinates optimised for different functionals are compared with the available low-temperature (15 K) experimental data [2] and the results of previous plane-wave PBE calculations [7] in Table 1. Comparing the values of the mean relative errors $\varepsilon_{l p}$ and $\varepsilon_{a n g}$ for the three calculated lattice parameters $(a, b, c)$ and angles $(\alpha$, $\beta, \gamma)$ relative to the experimental values, one can conclude that the LCAO method accurately reproduces the structural parameters of $\mathrm{CuWO}_{4}$. In fact, the LCAO results for all used functionals are closer to the low-temperature experimental data [2] than those of the plane-wave PBE calculations in [7]. For the LCAO method, the relative error is below $2 \%$ for pure DFT functionals, but it decreases below 1\% for the hybrid DFT-HF WCGGA-PBE-16\% and PBE0-13\%/25\% functionals.

The bulk modulus $B_{0}$ at zero pressure calculated from the second-order Murnaghan EOS for PBE (DFT-type) and PBE0-13\% (hybrid DFT-HFtype) functionals has consistent values of $77.0 \mathrm{GPa}$ and $84.2 \mathrm{GPa}$, respectively, which also agree with the value of $77 \mathrm{GPa}$ obtained in [7] from the plane-wave PBE calculations. However, these values are smaller than the experimental ones $B_{0}=134 \mathrm{GPa}$ and $171 \mathrm{GPa}$, obtained in [7] using silicone oil or argon as a pressure-transmitting medium.

\subsection{Local atomic structure}

Next, we will discuss the distortion of the local environment around copper and tungsten atoms caused by the FOJT [7, 8, 9] and SOJT [10] effects, respectively. Having different origins, the metal-oxygen octahedra also have different types of distortion. The FOJT effect results in the axial elongation of the $\mathrm{CuO}_{6}$ octahedra with four closest oxygen atoms located in an approx- 
imately square planar configuration and two oxygen atoms forming the axial $\mathrm{Cu}-\mathrm{O}$ bonds. At the same time, the SOJT effect leads to the out-of-centre displacement of the tungsten atoms towards the faces of the $\mathrm{WO}_{6}$ octahedra, which results in three short, two intermediate and one long $\mathrm{W}-\mathrm{O}$ distances.

Our LCAO calculations correctly predict a large distortion in both the $\mathrm{CuO}_{6}$ and the $\mathrm{WO}_{6}$ octahedra. While the results obtained for different hybrid DFT-HF functionals are close, the best agreement between the calculated distances and the low-temperature $(15 \mathrm{~K})$ experimental [2] inter-atomic distances has been found using the hybrid WCGGA-PBE-16\% functional (Table 2 and Fig. 2). Note that the axial FOJT distortion of the $\mathrm{CuO}_{6}$ octahedra is well reproduced: there are four oxygen atoms located in a plane at the distances $1.932 \AA, 1.955 \AA, 1.963 \AA$ and $1.987 \AA$, and the remaining two apical oxygen atoms are located at approximately $2.350 \AA$ and $2.409 \AA$. Furthermore, the Mulliken $\mathrm{Cu}-\mathrm{O}$ overlap population for apical oxygens is much smaller than that found for the four in-plane oxygen atoms, which indicates an anisotropy of $\mathrm{Cu}-\mathrm{O}$ bonding. As expected from the SOJT effect, the significant distortion in the $\mathrm{WO}_{6}$ octahedra is predicted by the LCAO results. The three nearest oxygen atoms are located at approximately 1.77-1.85 $\AA$, two oxygens at 1.97-2.04 $\AA$ and oxygen $\mathrm{O}(4)$ at approximately $2.15 \AA$. Note that the worst agreement is observed for the longest $R(\mathrm{~W}-\mathrm{O}(4))$ distance, but even in this case the difference is below $0.06 \AA$. The Mulliken $\mathrm{W}-\mathrm{O}$ overlap population is approximately $0.18-0.14$ for the three nearest oxygens and decreases to 0.04-0.08 for the three longest bonds.

To check the accuracy of the LCAO calculations in describing the local atomic structure of $\mathrm{CuWO}_{4}$, we have experimentally studied a distor- 
tion of the $\mathrm{CuO}_{6}$ and $\mathrm{WO}_{6}$ octahedra using X-ray absorption spectroscopy (XAS) at the $\mathrm{W} \mathrm{L}_{3}$ and $\mathrm{Cu} \mathrm{K}$ edges; thus, we were able to access both local environments independently. Because XAS is a local structure probe, it provides complementary information to the diffraction data $[39,40]$. In particular, the information on the radial distribution functions (RDFs) can be extracted from the extended X-ray absorption fine structure (EXAFS) using the regularisation technique $[41,42]$. This method allowed us to describe the octahedral distortion in terms of the metal-oxygen bond lengths distribution function [29].

In Fig. 2, we show the results obtained from analysing the ${\mathrm{W} \mathrm{L} 3^{-}}^{-}$and $\mathrm{Cu}$ K-edge EXAFS in polycrystalline $\mathrm{CuWO}_{4}$ at $10 \mathrm{~K}$, measured in transmission mode at HASYLAB/DESY synchrotron centre in Hamburg. A strong distortion of both metal octahedra in $\mathrm{CuWO}_{4}$, supplemented by thermal disorder effects, causes an overlap of contributions from the closely spaced $\mathrm{Cu}-\mathrm{O}$ and $\mathrm{W}-\mathrm{O}$ distances that makes it difficult to apply the conventional EXAFS analysis based on a sum of Gaussian-like components [43]. Therefore, we employed the regularisation-like method [41, 42], which allowed us to reconstruct the radial distribution functions (RDFs) $G(R)$ within the first coordination shell of tungsten and copper atoms (Fig. 2). The shape of both RDFs unambiguously confirms the strong distortion of metal-oxygen octahedra. One can clearly distinguish three peaks in the $\operatorname{RDF} G_{\mathrm{W}-\mathrm{O}}(R)$, centred at approximately $1.81 \AA, 2.02 \AA$ and $2.20 \AA$. These three peaks are due to 3, 2 and 1 oxygen atoms, respectively. For copper, there are only two peaks in the RDF $G_{\mathrm{Cu}-\mathrm{O}}(R)$ : the nearest group of four oxygen atoms in a plane is located at distances of approximately $1.97 \AA$, whereas the outer two oxygen 
atoms, which complete a distorted $\mathrm{CuO}_{6}$ octahedron, appear as a broad peak spreading from approximately $2.15 \AA$ to $2.56 \AA$. Thus, our EXAFS results agree with the low-temperature $(15 \mathrm{~K})$ neutron powder diffraction data from [2] and suggest the same type of octahedral distortion as obtained from our LCAO calculations.

\subsection{Electronic structure}

Although $\mathrm{CuWO}_{4}$ and $\mathrm{NiWO}_{4}$ have similar electronic structures [29], the presence of $\mathrm{Cu}^{2+}\left(3 d^{9}\right)$ ions in $\mathrm{CuWO}_{4}$ introduces a number of important differences between the two compounds.

Our LCAO calculations show that the unpaired electrons are well localised on the copper atoms, giving a net atomic spin of approximately 0.70 . The Mulliken population analysis was used to estimate the total atomic charges, which depend weakly on the functional type. The charges are equal to $+1.56 \mathrm{e}$ for $\mathrm{Cu},+2.76$ e for $\mathrm{W},-1.0$ e for $\mathrm{O}(1),-1.18$ e for $\mathrm{O}(2),-1.10$ e for $\mathrm{O}(3)$ and -1.04 e for $\mathrm{O}(4)$, which suggests a mixed ionic-covalent character of both $\mathrm{Cu}-$ $\mathrm{O}$ and $\mathrm{W}-\mathrm{O}$ bondings. In contrast to the purely ionic picture of a bare $\mathrm{W}^{6+}$ ion (with no valence electrons), its valence state in $\mathrm{CuWO}_{4}$ is approximately $3+$ due to back charge transfer from the nearest oxygen atoms. This fact leads to the second-order Jahn-Teller effect [44], which is responsible for a strong distortion of the $\mathrm{WO}_{6}$ octahedra.

The band structure diagram and the spin-dependent total/projected density of states (PDOS) for $\mathrm{CuWO}_{4}$ in the antiferromagnetic state are shown in Fig. 3 for the case of the hybrid DFT-HF PBE0-13\% functional, which is the best functional to reproduce the band gap value (Table 1). The dispersion curves are plotted along nine different symmetry directions in the 
Brillouin zone. According to our calculations, $\mathrm{CuWO}_{4}$ has an indirect band gap, which agrees with the experimental results of optical absorption spectroscopy $[19,45]$. The band gap values $E_{g}$ calculated for different functionals are given in Table 1. The DFT functionals underestimate the band gap value as expected. Note that our LCAO result $\left(E_{g}=0.54 \mathrm{eV}\right)$ and the result obtained using the spin-polarised FP-LAPW method [22] coincide when the same generalised-gradient approximation (GGA) is used for the exchangecorrelation potential. The two hybrid functionals PBE0-13\% and WCGGAPBE- $16 \%$ yield close values of $2.32 \mathrm{eV}$ and $2.56 \mathrm{eV}$, respectively, which are close to the experimental value of $2.3 \mathrm{eV}$ [19]. An increase of the HF admixture leads to an overestimated band gap value of $3.96 \mathrm{eV}$.

The main contribution to the band structure and spin-dependent DOS in $\mathrm{CuWO}_{4}$ originates from the $\mathrm{Cu} 3 d, \mathrm{~W} 5 d$ and $\mathrm{O} 2 p$ states (Fig. 3).

The valence band is approximately $7 \mathrm{eV}$ wide and is formed by the hybridised $\mathrm{O} 2 p, \mathrm{Cu} 3 d$ and $\mathrm{W} 5 d$ states. The $\mathrm{O} 2 p$ and $\mathrm{Cu} 3 d$ states dominate in the upper part of the valence band. In Fig. 4, we compare the PDOS curves in the range of the valence band, which was calculated using the PBE0-13\% functional, with the experimental valence-band X-ray photoelectron spectroscopy (XPS) spectra from [46]. The binding energy for the PDOS curves is given relative to the Fermi level placed at the top of the calculated valence band. The energy scale of the experimental XPS spectrum has been accordingly aligned. The valence-band XPS spectrum for $\mathrm{CuWO}_{4}$ shows a broad main peak with weakly resolved shoulder at the lower binding energies (around $+1 \mathrm{eV}$ in Fig. 4). Such shape correlates well with the theoretically calculated PDOS contributions that mainly come from the $\mathrm{O} 2 p$ and $\mathrm{Cu} 3 d$ 
states.

The bottom of the conduction band is composed mainly of empty $\mathrm{Cu} 3 d$ and $\mathrm{O} 2 p$ states, which are responsible for the narrow subband separated from the valence band by the band gap $E_{g}=2.3 \mathrm{eV}$. The empty W $5 d\left(t_{2 g}\right)$ states, hybridised with the $\mathrm{O} 2 p$ states, are separated from the $\mathrm{Cu}(3 d)-\mathrm{O}(2 p)$ subband by a gap of approximately $1 \mathrm{eV}$ and lie at approximately 4-6 eV above the top of the valence band. We found a different situation in $\mathrm{NiWO}_{4}$, where the bottom of the conduction band is formed by the empty W $5 d$ states with an admixture of the empty Ni $3 d$ states [29]. Note that in the related tungstate $\mathrm{ZnWO}_{4}$, the bottom of the conduction band is due to only the $\mathrm{W} 5 d$ and $\mathrm{O} 2 p$ states because the closed $3 d^{10}$ shell of $\mathrm{Zn}^{2+}$ ions creates the narrow well separated subband, observed at approximately -9 eV below the top of the valence band [28]. Thus, the type of transition metal ions in the tungstates influences significantly the nature of both the valence band and the conduction band.

The next group of higher-lying electronic states is again separated by a gap of approximately $1 \mathrm{eV}$ and starts at approximately $7 \mathrm{eV}$ above the top of the valence band. The bottom of this broad subband is dominated by the $\mathrm{W} 5 d\left(e_{g}\right)$ states mixed with $\mathrm{O} 2 p$ states.

The electronic band structure by LCAO qualitatively agrees with that obtained in [22] using the spin-polarised FP-LAPW method. Both methods predict a similar origin of the subbands, but their separation, i.e., the gap values, differ slightly. However, this difference can be mostly reduced by performing spin-polarised FP-LAPW calculations with the Hubbard-type Coulomb correction added in the framework of the so-called LDA $+U$ method 
$[22]$.

\subsection{Phonon structure}

According to group theoretical analysis, 36 phonon modes are expected for space group $P \overline{1}$ at the Brillouin-zone centre (the $\Gamma$-point) for $\mathrm{CuWO}_{4}$ with two formula units in the primitive unit cell. Half of them, 18 even $\mathrm{A}_{g}$ modes, are Raman-active, whereas the 18 odd $\mathrm{A}_{u}$ modes are infrared-active (three of them are acoustic modes with zero frequency at the $\Gamma$-point).

The calculated phonon frequencies are reported in Tables 3 and 4 for three hybrid functionals, which give the best agreement for the $\mathrm{CuWO}_{4}$ atomic and electronic structures as discussed above.

One can observe that the phonon frequencies are sensitive to the type of functional used in the calculations. In particular, the highest frequency, which corresponds to the $\mathrm{W}-\mathrm{O}$ vibration mode, increases upon mixing the HF contribution. Comparing the calculated frequencies for the Raman-active modes with the available experimental data from [7], one can conclude that the PBE0-13\% functional gives closest agreement. A similar result has been found by us in the case of $\mathrm{NiWO}_{4}$ in [29]. Note that the plane-wave PBE calculations [7] tend to underestimate the highest frequency stretching WO mode by approximately $50 \mathrm{~cm}^{-1}$. In fact, such underestimation appears typical for the plane-wave PBE scheme and was previously observed in other related tungstates: by $\sim 45 \mathrm{~cm}^{-1}$ in $\mathrm{ZnWO}_{4}$ [47] and by $\sim 32 \mathrm{~cm}^{-1}$ in $\mathrm{CdWO}_{4}$ [48]. We believe that the LCAO approach based on the local description of the electron wave functions can more accurately predict the local vibration frequencies when it is used in conjunction with the hybrid functionals and a properly optimised basis set. 


\section{Conclusions}

First-principle spin-polarised LCAO calculations have been performed for the triclinic $\mathrm{CuWO}_{4}$ using pure DFT and hybrid DFT/Hartree-Fock (HF) functionals. The atomic structure was fully relaxed for each functional, and the optimised atomic structure was used to calculate the energy band structure, the spin-dependent total and the projected density of electron states (DOS) and the phonon frequencies at the centre of the Brillouin zone (at the $\Gamma$-point).

Our total energy calculations for antiferromagnetic (AFM), ferromagnetic (FM) and non-magnetic (NM) orderings suggest that the AFM phase has the lowest energy, which agrees with the experimental results from $[2,6]$.

The band structure calculations using hybrid PBE0-13\% and WCGGAPBE-16\% functionals provide the band gap values $E_{g}=2.32 \mathrm{eV}$ and $2.56 \mathrm{eV}$, respectively, which agree with the experimental values $(2.0 \mathrm{eV}[11], 2.25 \mathrm{eV}$ $[12,18], 2.3 \mathrm{eV}[19])$. The calculated PDOS allowed us to interpret the atomic contributions into the electronic bands and show good agreement with the experimental valence-band XPS data from [46].

The optimised atomic structure calculated using the hybrid functionals reproduces well the low-temperature experimental data, which were obtained using diffraction in [2] and X-ray absorption spectroscopy (EXAFS) at the W $\mathrm{L}_{3}$ and $\mathrm{Cu} \mathrm{K}$ edges in the present work. The best agreement was found for the WCGGA-PBE-16\% functional, which presents a difference below $1 \%$ between the experimental and the calculated parameters. The strong distortions of both $\mathrm{CuO}_{6}$ and $\mathrm{WO}_{6}$ octahedra are explained by the mixed ionic-covalent character of both $\mathrm{Cu}-\mathrm{O}$ and $\mathrm{W}-\mathrm{O}$ bondings, leading to the first-order and 
second-order Jahn-Teller effects, respectively.

Finally, the phonon frequencies were calculated for both the Raman- and the infrared-active modes. The calculated Raman frequencies were compared with the available experimental data from [7], and the best agreement was found for the hybrid PBE0-13\% functional, which gives a difference of less than $20 \mathrm{~cm}^{-1}$.

To conclude, we have shown that the first-principle spin-polarised LCAO model based on a hybrid DFT-HF functional provides a good description of the properties of copper tungstate $\mathrm{CuWO}_{4}$. Moreover, the use of hybrid functionals is essential to obtain a quantitative agreement with the experiment.

\section{Acknowledgements}

This work was supported by the ESF Project 2009/0202/1DP/1.1.1.2.0/09/APIA/VIAA/141 Latvian Government Research Grant No. 09.1518 and Latvian National Research Program IMIS. The EXAFS experiments at HASYLAB/DESY have been supported from the European Community's Seventh Framework Programme (FP7/2007-2013) under grant agreement No. 226716 (project I20110160 EC).

\section{References}

[1] Anders AG, Zvyagin AI, Kobets MI, Pelikh LN, Khatsko EN, Yurko VG. Zh Eksp Theor Phys 1972;62:1798.

[2] Forsyth JB, Wilkinson C, Zvyagin AI. J Phys: Condens Matter $1991 ; 3: 8433$. 
[3] Lakey B, Tennanty DA, Cowleyz RA, Axez JD, Chen CK. J Phys: Condens Matter 1996;8:8613.

[4] Koo HJ, Whangbo MH. Inorg Chem 2001;40:2161.

[5] Schwarz B, Ehrenberg H, Weitzel H, Senyshyn A, Thybusch B, Knapp M, McIntyre GJ, Fuess H. Philos Mag 2008;88:1235.

[6] Weitzel H. Solid State Commun 1970;8:2071.

[7] Ruiz-Fuertes J, Errandonea D, Lacomba-Perales R, Segura A, González J, Rodríguez J, Manjón FJ, Ray S, Rodríguez-Hernández P, Muñoz A, Zhu Zh, Tu CY. Phys Rev B 2010;81:224115.

[8] Ruiz-Fuertes J, Friedrich A, Pellicer-Porres J, Errandonea D, Segura A, Morgenroth W, Haussühl E, Tu CY, Polian A. Chem Mater 2011;23:4220.

[9] Ruiz-Fuertes J, Segura A, Rodríguez F, Errandonea D, Sanz-Ortiz MN. Phys Rev Lett 2012;108:166402.

[10] Kunz M, Brown ID. J Solid State Chem 1995;115:395.

[11] Pandey PK, Bhave NS, Kharat RB. Mater Lett 2005;59:3149.

[12] Chang Y, Braun A, Deangelis A, Kaneshiro J, Gaillard N. J Phys Chem C 2011;115:25490.

[13] Li CL, Fu ZW. Electrochim Acta 2008;53:4293.

[14] Schmitt P, Brem N, Schunk S, Feldmann C. Adv Funct Mater $2011 ; 21: 3037$. 
[15] Selvan RK, Gedanken A. Nanotechnol 2009;20:105602.

[16] Naik SJ, Salker AV. Catal Commun 2009;10:884.

[17] Dusastre V, Williams DE. J Mater Chem 1999;9:965.

[18] Yourey JE, Bartlett BM. J Mater Chem 2011;21:7651.

[19] Ruiz-Fuertes J, Errandonea D, Segura A, Manjn FJ, Zhu Zh, Tu CY, High Pressure Res 2008;28:565.

[20] Khyzhun OYu, Bekenev VL, Solonin YuM. J Alloys Compd 2009;480:184.

[21] Atuchin VV, Troitskaia IB, Khyzhun OYu, Bekenev VL, Solonin YuM. Int J Appl Phys Math 2011;1:19.

[22] Lalić MV, Popović ZS, Vukajlović FR. Comput Mater Sci 2011;50:1179.

[23] Evarestov RA. Quantum Chemistry of Solids. Berlin: Springer; 2007.

[24] Dovesi R, Saunders VR, Roetti C, Orlando R, Zicovich-Wilson CM, Pascale F, Civalleri B, Doll K, Harrison NM, Bush IJ, D'Arco P, Llunell M. CRYSTAL09 User's Manual. Torino: University of Torino; 2009.

[25] Evarestov RA, Kalinko A, Kuzmin A, Losev M, Purans J. Integr Ferroelectr 2009;108:1.

[26] Ruiz E, Llunell M, Alemany P, J Solid State Chem 2003;176:400.

[27] Piskunov S, Heifets E, Eglitis RI, Borstel G. Comput Mater Sci 2004;29:165. 
[28] Kalinko A, Kuzmin A, Evarestov RA, Solid State Commun 2009;149:425.

[29] Kuzmin A, Kalinko A, Evarestov RA, Centr Eur J Phys 2011;9:502.

[30] Monkhorst HJ, Pack JD. Phys Rev B 1976;13:5188.

[31] Vosko SH, Wilk L, Nusair M. Can J Phys 1980;58:1200.

[32] Perdew JP, Chevary JA, Vosko SH, Jackson KA, Pederson MR, Singh DJ, Fiolhais C, Phys Rev B 1992;46:6671.

[33] Perdew JP, Burke K, Ernzerhof M. Phys Rev Lett 1996;77:3865.

[34] Adamo C, Barone V. J Chem Phys 1999;110:6158.

[35] Wu Zh, Cohen RE. Phys Rev B 2006;73:235116.

[36] Perger WF, Criswell J, Civalleri B, Dovesi R. Comp Phys Commun 2009;180:1753.

[37] Pascale F, Zicovich-Wilson CM, Lopez F, Civalleri B, Orlando R, Dovesi R. J Comput Chem 2004;25:888.

[38] Kihlborg L, Gebert E. Acta Cryst B 1970;26:1020.

[39] Rehr JJ, Albers RC. Rev Mod Phys 2000;72:621.

[40] Aksenov VL, Kuzmin AYu, Purans J, Tyutyunnikov SI. Phys Part Nucl 2001;32:675.

[41] Kuzmin A. J Physique IV (France) 1997;7:C2-213. 
[42] Kuzmin A, Purans J. J Phys: Condens Matter 2000;12:1959.

[43] Ruiz-Fuertes J, Sanz-Ortiz MN, González J, Rodríguez F, Segura A, Errandonea D. J Phys: Conf Ser 2010;215:012048.

[44] Walkingshaw AD, Spaldin NA, Artacho E. Phys Rev B 2004;70:165110.

[45] Lacomba-Perales R, Ruiz-Fuertes J, Errandonea D, Martínez-García D, Segura A, Europhys Lett 2008;83:37002.

[46] Khyzhun OYu, Strunskus T, Cramm S, Solonin YuM. J Alloys Compd 2005;389:14.

[47] Errandonea D, Manjón FJ, Garro N, Rodríguez-Hernández P, Radescu S, Mujica A, Muñoz A, Tu CY. Phys Rev B 2008;78:054116.

[48] Lacomba-Perales R, Errandonea D, Martínez-García D, RodríguezHernández P, Radescu S, Mujica A, Muñoz A, Chervin JC, Polian A. Phys Rev B 2009;79:094105. 

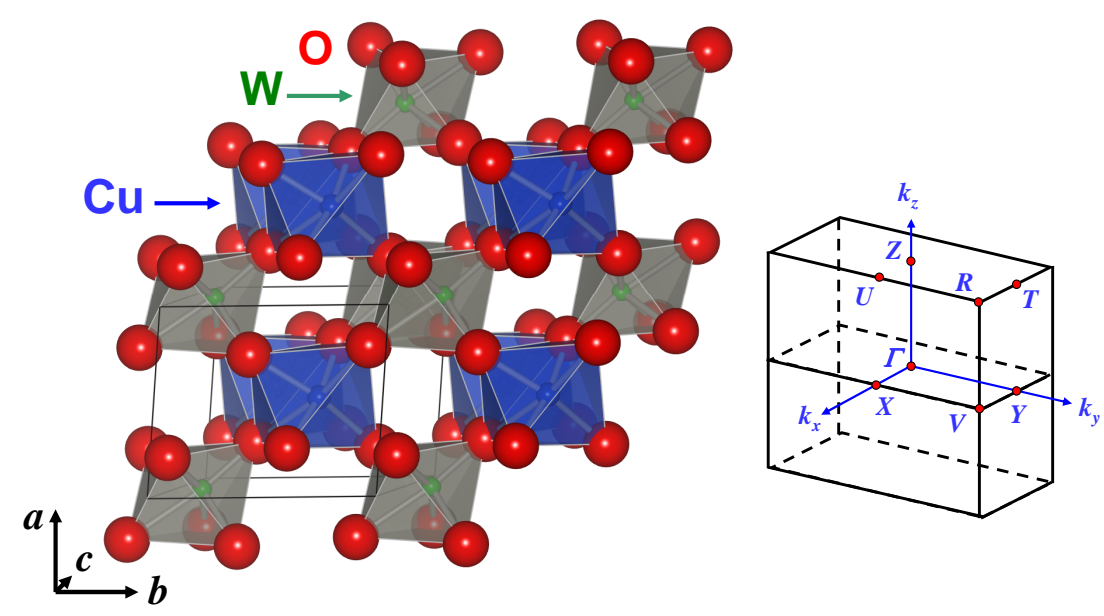

Figure 1: (colour online) Crystal structure and the first Brillouin zone of the triclinic $\mathrm{CuWO}_{4}$ (space group $P \overline{1}$ (No. 2)). 

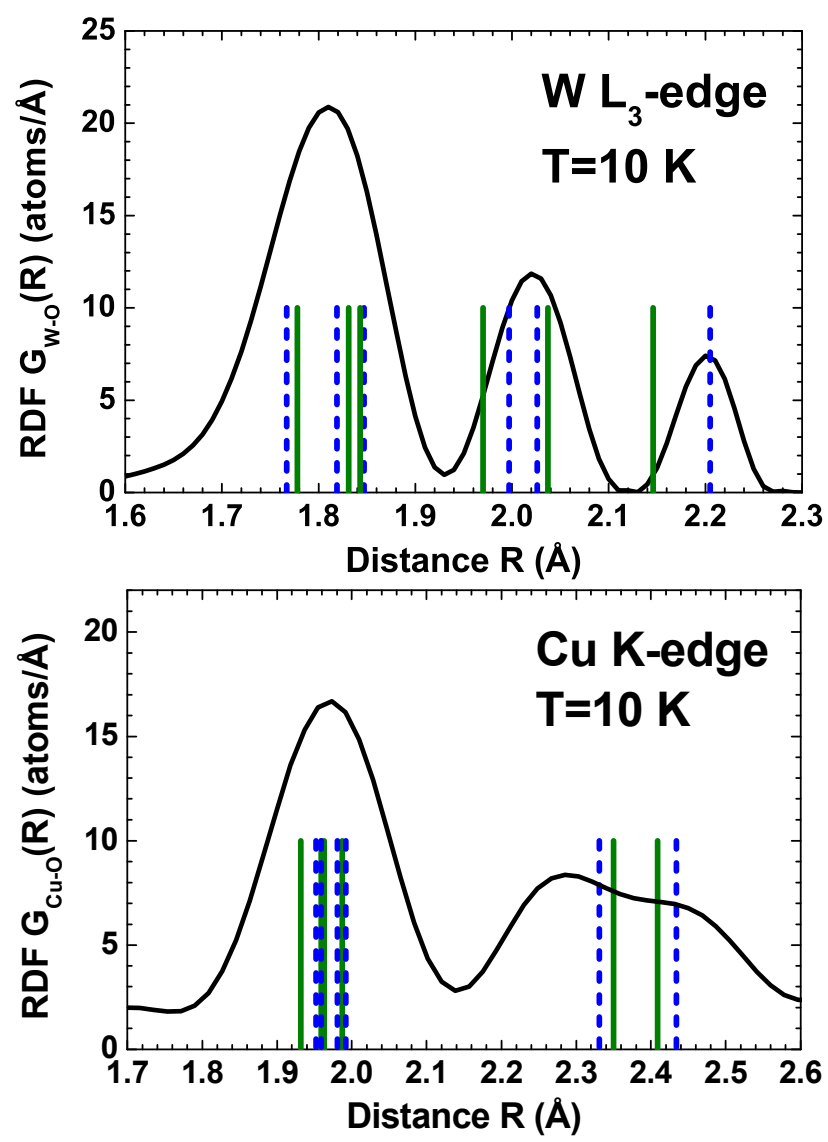

Figure 2: (colour online) Comparison of the radial distribution functions (RDFs) for the first coordination shell around tungsten and copper, obtained from the analysis of the $\mathrm{W} \mathrm{L}_{3^{-}}$and $\mathrm{Cu} \mathrm{K}$-edge EXAFS spectra at $10 \mathrm{~K}$ (solid curves), with the $\mathrm{W}-\mathrm{O} / \mathrm{Cu}-\mathrm{O}$ bond lengths obtained using neutron powder diffraction in [2] at $15 \mathrm{~K}$ (dashed vertical lines) and the spin-dependent LCAO calculations (solid vertical lines) in the present work. The LCAO calculations were performed using the hybrid DFT-HF WCGGA-PBE-16\% functional for the antiferromagnetic (AFM) state. 

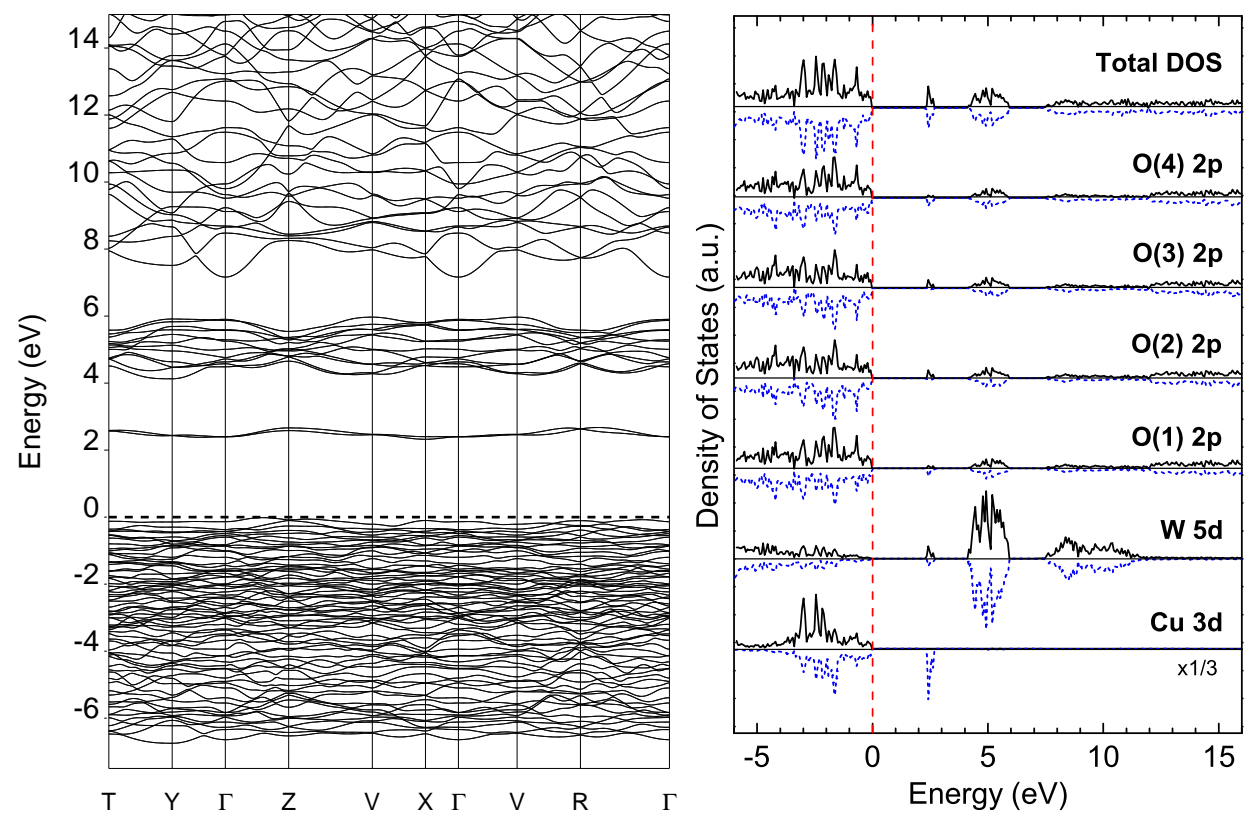

Figure 3: (colour online) Band structure diagram (left panel) and spin-dependent total/projected density of states (right panel) for $\mathrm{CuWO}_{4}$ as calculated using the firstprinciple LCAO method with the hybrid DFT-HF PBE0-13\% functional. The energy zero is set at the top of the valence band (Fermi energy position). Contributions of majority and minority spin states to the total DOS are shown by solid (black) and dashed (blue) lines, respectively. 


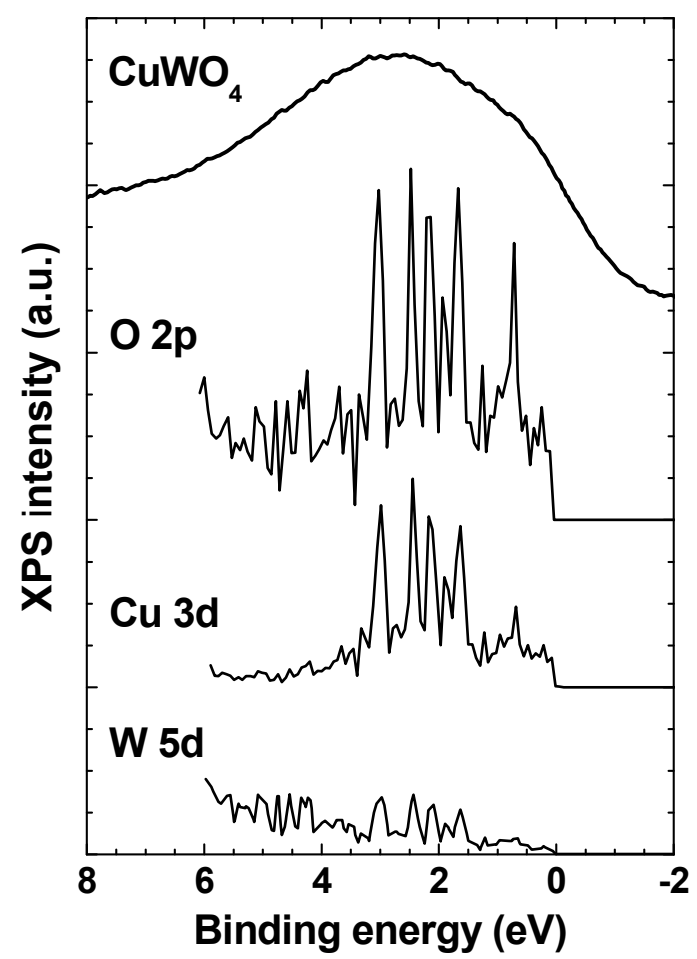

Figure 4: Comparison of the experimental valence-band XPS spectrum from [46] and PDOS curves (from Fig. 3) for $\mathrm{CuWO}_{4}$. The curves are vertically shifted for clarity. The experimental spectrum corresponds to the spectrum excited by photons with a $200 \mathrm{eV}$ energy and recorded with a pass energy of $20 \mathrm{eV}$ in [46]. 
Table 1: Comparison of the lattice parameters $(a, b, c, \alpha, \beta, \gamma)$ and the atomic fractional coordinates $(x, y, z)$, which were obtained using spin-polarised LCAO calculations for the antiferromagnetic (AFM) state, with the low-temperature (15 K) neutron powder diffraction data from $[2]$ for $\mathrm{CuWO}_{4}$ (space group $P \overline{1}$ ). $\varepsilon_{l p}$ and $\varepsilon_{a n g}$ are the mean relative errors for the three calculated lattice parameters $(a, b, c)$ and angles $(\alpha, \beta, \gamma)$ relative to the experimental values. $V_{0}$ is the unit cell volume. The bulk modulus $B_{0}$ (GPa) and its first pressure derivative $B_{0}^{\prime}$ (dimensionless) were calculated from the second-order Murnaghan equation. The experimental value of the indirect band gap $E_{\mathrm{g}}$ was taken from [19].

\begin{tabular}{|c|c|c|c|c|c|c|c|c|}
\hline & $\begin{array}{c}\text { Exp. }[2] \\
(T=15 \mathrm{~K})\end{array}$ & $\mathrm{PBE}[7]$ & LDA & PWGGA & $\mathrm{PBE}$ & PBE0-13\% & $\begin{array}{l}\text { WCGGA- } \\
\text { PBE- } 16 \%\end{array}$ & PBE0-25\% \\
\hline$\overline{a(\AA)}$ & 4.694 & 4.8352 & 4.595 & 4.769 & 4.782 & 4.766 & 4.686 & 4.742 \\
\hline$b(\AA)$ & 5.830 & 6.0538 & 5.713 & 5.925 & 5.939 & 5.907 & 5.796 & 5.863 \\
\hline$c(\AA)$ & 4.877 & 4.9429 & 4.865 & 4.944 & 4.946 & 4.916 & 4.861 & 4.890 \\
\hline$\varepsilon_{l p}(\%)$ & & 2.73 & 1.45 & 1.53 & 1.72 & 1.22 & 0.36 & 0.62 \\
\hline$\alpha\left({ }^{\circ}\right)$ & 91.64 & 93.26 & 92.46 & 92.78 & 92.75 & 92.45 & 92.20 & 92.18 \\
\hline$\beta\left(^{\circ}\right)$ & 92.41 & 94.25 & 92.16 & 93.43 & 93.48 & 93.35 & 92.61 & 93.07 \\
\hline$\gamma\left({ }^{\circ}\right)$ & 82.91 & 80.11 & 84.80 & 82.41 & 82.25 & 82.22 & 83.44 & 82.71 \\
\hline$\varepsilon_{a n g}(\%)$ & & 2.38 & 1.15 & 0.98 & 1.05 & 0.91 & 0.49 & 0.52 \\
\hline $\mathrm{Cu}(x)$ & 0.49537 & 0.7523 & 0.4962 & 0.4928 & 0.4923 & 0.4933 & 0.4951 & 0.4950 \\
\hline $\mathrm{Cu}(y)$ & 0.65942 & 0.5662 & 0.6579 & 0.6567 & 0.6568 & 0.6576 & 0.6583 & 0.6598 \\
\hline $\mathrm{Cu}(z)$ & 0.24524 & 0.6569 & 0.2482 & 0.2490 & 0.2489 & 0.2474 & 0.2468 & 0.2463 \\
\hline $\mathrm{W}(x)$ & 0.02146 & 0.7899 & 0.0112 & 0.0176 & 0.0184 & 0.0188 & 0.0154 & 0.0170 \\
\hline $\mathrm{W}(y)$ & 0.17353 & 0.5705 & 0.1745 & 0.1717 & 0.1717 & 0.1719 & 0.1740 & 0.1729 \\
\hline $\mathrm{W}(z)$ & 0.25405 & 0.1228 & 0.2528 & 0.2547 & 0.2549 & 0.2553 & 0.2540 & 0.2551 \\
\hline $\mathrm{O}(1)(x)$ & 0.25042 & 0.2663 & 0.2586 & 0.2525 & 0.2525 & 0.2505 & 0.2535 & 0.2505 \\
\hline $\mathrm{O}(1)(y)$ & 0.35475 & 0.9023 & 0.3615 & 0.3512 & 0.4285 & 0.3500 & 0.3548 & 0.3510 \\
\hline $\mathrm{O}(1)(z)$ & 0.42520 & 0.6204 & 0.4269 & 0.4285 & 0.4285 & 0.4283 & 0.4266 & 0.4267 \\
\hline $\mathrm{O}(2)(x)$ & 0.21551 & 0.2150 & 0.2176 & 0.2108 & 0.2103 & 0.2114 & 0.2142 & 0.2121 \\
\hline $\mathrm{O}(2)(y)$ & 0.88077 & 0.9538 & 0.8827 & 0.8802 & 0.8802 & 0.8805 & 0.8818 & 0.8816 \\
\hline $\mathrm{O}(2)(z)$ & 0.42901 & 0.0813 & 0.4278 & 0.4308 & 0.4313 & 0.4317 & 0.4305 & 0.4321 \\
\hline $\mathrm{O}(3)(x)$ & 0.73511 & 0.5095 & 0.7360 & 0.7373 & 0.7378 & 0.7367 & 0.7371 & 0.7362 \\
\hline $\mathrm{O}(3)(y)$ & 0.38077 & 0.7492 & 0.3855 & 0.3819 & 0.3820 & 0.3812 & 0.3833 & 0.3805 \\
\hline $\mathrm{O}(3)(z)$ & 0.09822 & 0.3471 & 0.0911 & 0.0954 & 0.0955 & 0.0967 & 0.0940 & 0.0971 \\
\hline $\mathrm{O}(4)(x)$ & 0.78214 & 0.9777 & 0.7775 & 0.7833 & 0.7837 & 0.7853 & 0.7833 & 0.7865 \\
\hline $\mathrm{O}(4)(y)$ & 0.90774 & 0.7434 & 0.9085 & 0.9145 & 0.9151 & 0.9139 & 0.9115 & 0.9118 \\
\hline $\mathrm{O}(4)(z)$ & 0.05459 & 0.8318 & 0.0591 & 0.0495 & 0.0488 & 0.0488 & 0.0547 & 0.0501 \\
\hline$V_{0}\left(\AA^{3}\right)$ & 132.3 & 142.0 & 127.0 & 138.1 & 138.8 & 136.8 & 131.0 & 134.6 \\
\hline$B_{0}(\mathrm{GPa})$ & $\begin{array}{l}134[7] \\
171[7]\end{array}$ & 77 & & & 77.0 & 84.2 & & \\
\hline$B_{0}^{\prime}$ & & 4 & & & 11.1 & 11.2 & & \\
\hline$E_{g}(\mathrm{eV})$ & $2.3[19]$ & & 0.06 & $25 \quad 0.54$ & 0.54 & 2.32 & 2.56 & 3.96 \\
\hline
\end{tabular}


Table 2: $R(\mathrm{Cu}-\mathrm{O})$ and $R(\mathrm{~W}-\mathrm{O})$ bond lengths $(\AA)$ within the $\mathrm{CuO}_{6}$ and $\mathrm{WO}_{6}$ octahedra, calculated from the low-temperature $(15 \mathrm{~K})$ diffraction data [2] and from a set of structural parameters obtained by the LCAO method using the hybrid DFT-HF WCGGA-PBE-16\% functional (Table 1). $\langle R(\mathrm{Cu}-\mathrm{O})\rangle$ and $\langle R(\mathrm{~W}-\mathrm{O})\rangle$ are the mean values $(\AA)$ of the bond lengths, $\sigma(\mathrm{Cu}-\mathrm{O})$ and $\sigma(\mathrm{W}-\mathrm{O})$ are their standard deviations $(\AA) . \Delta$ is the difference $(\AA)$ between the experimental results and the LCAO calculation results.

\begin{tabular}{|c|c|c|c|}
\hline & $\begin{array}{c}\text { Exp. [2] } \\
(15 \mathrm{~K})\end{array}$ & $\begin{array}{c}\text { AFM LCAO } \\
\text { WCGGA-PBE- } 16 \%\end{array}$ & $\Delta$ \\
\hline$R(\mathrm{Cu}-\mathrm{O}(2))$ & 1.952 & 1.955 & -0.003 \\
\hline$R(\mathrm{Cu}-\mathrm{O}(1))$ & 1.959 & 1.932 & 0.027 \\
\hline$R(\mathrm{Cu}-\mathrm{O}(3))$ & 1.980 & 1.963 & 0.017 \\
\hline$R(\mathrm{Cu}-\mathrm{O}(3))$ & 1.992 & 1.987 & 0.005 \\
\hline$R(\mathrm{Cu}-\mathrm{O}(4))$ & 2.331 & 2.350 & -0.019 \\
\hline$R(\mathrm{Cu}-\mathrm{O}(1))$ & 2.434 & 2.409 & 0.025 \\
\hline$\langle R(\mathrm{Cu}-\mathrm{O})\rangle$ & 2.108 & 2.099 & 0.009 \\
\hline$\sigma(\mathrm{Cu}-\mathrm{O})$ & 0.197 & 0.199 & -0.003 \\
\hline$R(\mathrm{~W}-\mathrm{O}(1))$ & 1.767 & 1.778 & -0.011 \\
\hline$R(\mathrm{~W}-\mathrm{O}(4))$ & 1.819 & 1.831 & -0.012 \\
\hline$R(\mathrm{~W}-\mathrm{O}(3))$ & 1.847 & 1.843 & 0.004 \\
\hline$R(\mathrm{~W}-\mathrm{O}(2))$ & 1.997 & 1.974 & 0.023 \\
\hline$R(\mathrm{~W}-\mathrm{O}(2))$ & 2.026 & 2.039 & -0.013 \\
\hline$R(\mathrm{~W}-\mathrm{O}(4))$ & 2.205 & 2.146 & 0.059 \\
\hline$\langle R(\mathrm{~W}-\mathrm{O})\rangle$ & 1.944 & 1.935 & 0.008 \\
\hline$\sigma(\mathrm{W}-\mathrm{O})$ & 0.149 & 0.130 & 0.019 \\
\hline
\end{tabular}


Table 3: Comparison of the different Raman scattering modes $\left(\mathrm{cm}^{-1}\right)$ in $\mathrm{CuWO}_{4}$ (space group $P \overline{1})$ : the experimentally obtained modes in $[7]$ and the theoretically calculated modes from the first-principles using the plane-wave PBE method in [7] and by the LCAO method in the present work for the antiferromagnetic (AFM) state with three hybrid DFT-HF functionals.




Table 4: Comparison of the different infrared modes $\left(\mathrm{cm}^{-1}\right)$ in $\mathrm{CuWO}_{4}$ (space group $\left.P \overline{1}\right)$ : the theoretically calculated modes from the first-principles using the plane-wave PBE method in [7] and the modes obtained using the LCAO method in the present work for the antiferromagnetic (AFM) state with three hybrid DFT-HF functionals.

\begin{tabular}{|c|c|c|c|c|}
\hline & AFM PW [7] & AFM LCAO & AFM LCAO & AFM LCAO \\
\hline & $\mathrm{PBE}$ & PBE0-13\% & WCGGA-PBE-16\% & PBE0-25\% \\
\hline $\mathrm{A}_{u}$ & 0 & 0 & 0 & 0 \\
\hline $\mathrm{A}_{u}$ & 0 & 0 & 0 & 0 \\
\hline $\mathrm{A}_{u}$ & 0 & 0 & 0 & 0 \\
\hline $\mathrm{A}_{u}$ & 101.4 & 125 & 133 & 129 \\
\hline $\mathrm{A}_{u}$ & 157.3 & 173 & 181 & 180 \\
\hline $\mathrm{A}_{u}$ & 214.2 & 230 & 234 & 235 \\
\hline $\mathrm{A}_{u}$ & 239.0 & 264 & 277 & 271 \\
\hline $\mathrm{A}_{u}$ & 266.1 & 285 & 282 & 290 \\
\hline $\mathrm{A}_{u}$ & 281.0 & 300 & 309 & 309 \\
\hline $\mathrm{A}_{u}$ & 320.5 & 344 & 357 & 350 \\
\hline $\mathrm{A}_{u}$ & 332.3 & 363 & 376 & 375 \\
\hline $\mathrm{A}_{u}$ & 383.8 & 420 & 438 & 432 \\
\hline $\mathrm{A}_{u}$ & 438.9 & 463 & 478 & 476 \\
\hline $\mathrm{A}_{u}$ & 474.8 & 540 & 558 & 556 \\
\hline $\mathrm{A}_{u}$ & 516.5 & 548 & 571 & 564 \\
\hline $\mathrm{A}_{u}$ & 639.6 & 679 & 698 & 695 \\
\hline $\mathrm{A}_{u}$ & 727.7 & 762 & 775 & 779 \\
\hline $\mathrm{A}_{u}$ & 852.7 & 899 & 912 & 927 \\
\hline
\end{tabular}

